\title{
DIFFUSION EQUATION INCLUDING A LOCAL FRACTIONAL DERIVATIVE AND WEIGHTED INNER PRODUCT
}

\author{
Suleyman Cetinkaya, Ali Demir \\ Faculty of Arts and Sciences, Kocaeli University \\ Kocaeli, Turkey \\ suleyman.cetinkaya@kocaeli.edu.tr,ademir@kocaeli.edu.tr
}

Received: 15 June 2021; Accepted: 11 August 2021

\begin{abstract}
In this research, we discuss the construction of the analytic solution of the homogenous initial boundary value problem including partial differential equations of fractional order. Since the homogenous initial boundary value problem involves a local fractional order derivative, it has classical initial and boundary conditions. By means of separation of the variables method and the inner product defined on $L^{2}[0, l]$, the solution is constructed in the form of a Fourier series including the exponential function. The illustrative examples present the applicability and influence of the separation of variables method on time fractional diffusion problems. Moreover, as the fractional order $\alpha$ tends to 1 , the solution of the fractional diffusion problem tends to the solution of the diffusion problem which proves the accuracy of the solution.
\end{abstract}

MSC 2010: 26A33, 65M70

Keywords: local fractional derivative, Dirichlet boundary conditions, spectral method, separation of variables, weighted inner product

\section{Introduction}

Since mathematical models including fractional derivatives play a vital role fractional derivatives, they attract a growing attention of many researchers in various branches of sciences. Therefore, there are many different fractional derivatives such as the Caputo, Riemann-Liouville, and Atangana-Baleanu. However, these fractional derivatives do not satisfy the most important properties of the ordinary derivative which leads to many difficulties in analyzing and obtaining the solutions of fractional mathematical models. As a result, many scientists focus on defining new fractional derivatives to cover the setbacks of the defined ones. Moreover, the success of mathematical modelling of systems or processes depends on the fractional derivative it involves, since the correct choice of the fractional derivative allows us to model the real data of systems or processes accurately.

In order to the define new fractional derivatives, various methods exist and these are classified based on their features and formation such as nonlocal fractional deriva- 
tives and local fractional derivatives. The proportional derivative is a newly defined fractional derivative which is generally defined as

$$
{ }^{P} D_{\alpha} f(t)=K_{1}(\alpha, t) f(t)+K_{0}(\alpha, t) f^{\prime}(t),
$$

where the functions $K_{0}$ and $K_{1}$ satisfy certain properties in terms of limit [1], and $f$ is a differentiable function. Notice that this derivative can be regarded as an extension of a conformable derivative and is used in control theory.

In this study we focus on obtaining the solution of the following fractional diffusion equation including various proportional derivative operators by making use of the separation of variables method:

$$
\begin{aligned}
& { }^{P} D_{t}^{\alpha} u(x, t)=u_{x x}(x, t)+B u_{x}(x, t)-C u(x, t ;), \\
& u(0, t)=u(l, t ;)=0, \\
& u(x, 0)=f(x) e^{-\frac{B}{2} x}
\end{aligned}
$$

where $0<\alpha<1,0 \leq x \leq l, 0 \leq t \leq T, B, C \in \mathbb{R}$. Here we use the following forms of the proportional derivatives:

$$
{ }^{P} D_{\alpha} f(t)=K_{1}(\alpha) f(t)+K_{0}(\alpha) f^{\prime}(t) .
$$

We especially consider the following ones:

$$
{ }_{1}^{P} D_{\alpha} f(t)=(1-\alpha) f(t)+\alpha f^{\prime}(t)
$$

and

$$
{ }_{2}^{P} D_{\alpha} f(t)=\left(1-\alpha^{2}\right) f(t)+\alpha^{2} f^{\prime}(t) .
$$

From a physical perspective, the intrinsic nature of the physical system can be reflected to the mathematical model of the system by using fractional derivatives. Therefore the solution of the fractional mathematical model is in excellent agreement with the predictions and experimental measurement of it. The systems whose behaviour is non-local can be modelled better by fractional mathematical models, and the degree of its non-locality can be arranged by the order of a fractional derivative. In order to analyze the diffusion in a non-homogenous medium that has memory effects, it is better to analyze the solution of the fractional mathematical model for this diffusion. As a result, in order to model a process, the correct choices of a fractional derivative and its order must be determined.

In this study, the local fractional derivative is used to model diffusion problems, since models including local fractional derivatives gives better results than models including integer order derivatives. In the mathematical modelling of a diffusion problem for different matters such as liquid, gas and temperature, the suitable fractional order $\alpha$ is chosen, since the diffusion coefficient $\gamma^{2}$ depends on the order $\alpha$ of a fractional derivative [2]. This mathematical modelling describes the behaviour of matter 
in a phase. There are many published works on the diffusion of various matters in science, especially in fluid mechanics and gas dynamics [3-20]. From this aspect, analysis of this problem plays an important role in application. Moreover, sub-diffusion cases for which $0<\alpha<1$ are under consideration. The solution of the fractional mathematical model of sub-diffusion cases behaves much slower than the solution of the integer-order mathematical model unlike the fractional mathematical model for super-diffusion.

\section{Main results}

Let us consider the following problem including the proportional derivative in (6)

$$
\begin{aligned}
& { }^{P} D_{t}^{\alpha} u(x, t)=u_{x x}(x, t)+B u_{x}(x, t)-C u(x, t ;), \\
& u(0, t)=u(l, t ;)=0, \\
& u(x, 0)=f(x) e^{-\frac{B}{2} x}
\end{aligned}
$$

where $0<\alpha<1,0 \leq x \leq l, 0 \leq t \leq T, B, C \in \mathbb{R}$. The analytic form of the solution for the problem (8)-(10) is established by employing the well known separation of variables method.

$$
u(x, t ; \alpha)=X(x) T(t ; \alpha)
$$

where $0 \leq x \leq l, 0 \leq t \leq T$.

Utilizing (11) in (8) and rearranging them leads to the following:

$$
\frac{{ }^{P} D_{t}^{\alpha}(T(t ; \alpha))}{T(t ; \alpha)}+C=\frac{X^{\prime \prime}(x)+B X^{\prime}(x)}{X(x)}=-\lambda^{2} .
$$

Taking the right hand side of equation (12) and related boundary conditions (9) into account, the following problem is obtained:

$$
\begin{aligned}
& X^{\prime \prime}(x)+B X^{\prime}(x)+\lambda X(x)=0, \\
& X(0)=X(l)=0 .
\end{aligned}
$$

which has the solution $X(x)=e^{r x}$. As a result, the following characteristic equation is reached $r^{2}+B r+\lambda=0$.

Case 1. If $B^{2}-4 \lambda>0$, the solutions of the characteristic equation are two distinct real roots $r_{1} \neq r_{2}$ which allows us to obtain the solution of the problem (13)-(14) as $X(x)=c_{1} e^{r_{1} x}+c_{2} e^{r_{2} x}$. The first boundary condition yields $X(0)=0=c_{1}+c_{2} \Longrightarrow$ $c_{1}=-c_{2}$. Similarly, the second boundary condition leads to $X(l)=c_{1}\left(e^{r_{1} l}-e^{r_{2} l}\right)=$ $0 \Longrightarrow c_{1}=0, c_{2}=0$ which implies that $X(x)=0$ which implies that there is no solution for $B^{2}-4 \lambda>0$.

Case 2. If $B^{2}-4 \lambda=0$, the solutions of the characteristic equation are two coincident real roots $r_{1}, r_{2}$ which leads to the solution of the problem (13)-(14) as 
$X(x)=c_{1} e^{r_{1} x}+c_{1} e^{r_{2} x}$. By making use of the first boundary condition, we have $X(0)=c_{1}=0$. Similarly, the second boundary condition leads to $X(l)=c_{2} l e^{r_{1} l} \Longrightarrow$ $c_{2}=0$ which implies that $X(x)=0$ which implies that there is no solution for $B^{2}-4 \lambda=0$.

Case 3. If $B^{2}-4 \lambda<0$, the solutions of the characteristic equation are two complex roots which leads to the solution of the problem (13)-(14) as

$X(x)=e^{-\frac{B}{2} x}\left(k_{1} \cos \left(\frac{\sqrt{4 \lambda-B^{2}}}{2} x\right)+i k_{2} \sin \left(\frac{\sqrt{4 \lambda-B^{2}}}{2} x\right)\right)$. By making use of the first boundary condition, we have $X(0)=k_{1}=0$. Similarly, the last boundary condition leads to $X(l)=e^{-\frac{B}{2} l} i k_{2} \sin \left(\frac{\sqrt{4 \lambda-B^{2}}}{2} l\right)=0$, which implies that $\sin \left(\frac{\sqrt{4 \lambda-B^{2}}}{2} l\right)=0$, which yields the following eigenvalues

$$
\lambda_{n}(\beta)=\frac{4 w_{n}^{2}+B^{2} l^{2}}{4 l^{2}}=\frac{4 w_{n}^{2}+(B l)^{2}}{(2 l)^{2}}, n=0,1,2,3, \ldots, \lambda_{1}<\lambda_{2}<\lambda_{3}<\ldots
$$

where $w_{n}=n \pi, n=0,1,2,3, \ldots$ satisfy the equation $\sin \left(w_{n}\right)=\sin \left(\frac{\sqrt{4 \lambda_{n}-B^{2}}}{2} l\right)=0$.

As a result, the solution is obtained as follows:

$$
X_{n}(x)=\sin \left(w_{n}\left(\frac{x}{l}\right)\right) e^{-\frac{B}{2} x}, n=1,2,3, \ldots
$$

The second equation in (12) for eigenvalue $\lambda_{n}$ yields the ordinary differential equation below:

$$
\begin{aligned}
& \frac{{ }^{P} D_{t}^{\alpha}(T(t ; \alpha))}{T(t ; \alpha)}=-(C+\lambda), \\
& \frac{K_{1}(\alpha) T_{n}(t ; \alpha)+K_{0}(\alpha) T_{n}^{\prime}(t ; \alpha)}{T_{n}(t ; \alpha)}=-(C+\lambda), \\
& K_{0}(\alpha) T_{n}^{\prime}(t ; \alpha)+\left(C+\lambda+K_{1}(\alpha)\right) T_{n}(t ; \alpha)=0,
\end{aligned}
$$

which yields the following solution

$$
T_{n}(t ; \alpha)=\exp \left(-\frac{C+\lambda+K_{1}(\alpha)}{K_{0}(\alpha)} t\right), n=0,1,2,3, \ldots
$$

The solution for every eigenvalue $\lambda_{n}$ is constructed as

$$
\begin{aligned}
u_{n}(x, t ; \alpha) & =X_{n}(x) T_{n}(t ; \alpha) \\
& =\exp \left(-\frac{C+\lambda+K_{1}(\alpha)}{K_{0}(\alpha)} t\right) \sin \left(w_{n}\left(\frac{x}{l}\right)\right) e^{-\frac{B}{2} x}, n=0,1,2,3, \ldots
\end{aligned}
$$


which leads to the following general solution

$$
u(x, t ; \alpha)=\sum_{n=1}^{\infty} A_{n} e^{-\frac{B}{2} x} \sin \left(w_{n}\left(\frac{x}{l}\right)\right) \exp \left(-\frac{C+\lambda+K_{1}(\alpha)}{K_{0}(\alpha)} t\right) .
$$

Note that it satisfies the boundary condition and fractional differential equation.

The coefficients of the general solution are established by taking the following initial condition into account:

$$
u(x, 0)=f(x) e^{-\frac{B}{2} x}=\sum_{n=1}^{\infty} A_{n} e^{-\frac{B}{2} x} \sin \left(w_{n}\left(\frac{x}{l}\right)\right) .
$$

The coefficients $A_{n}$ for $n=1,2,3, \ldots$ determined by the help of inner product with a weighted function defined on $L^{2}[0, l]$ as $\left\langle u, v>=\frac{2}{l} \int_{0}^{l} u(x) v(x) e^{B x} d x\right.$ :

$$
\begin{aligned}
A_{n} & =\frac{2}{l}<e^{-\frac{B}{2} x} \sin \left(w_{n}\left(\frac{x}{l}\right)\right), f(x) e^{-\frac{B}{2} x}>. \\
& =\frac{2}{l} \int_{0}^{l} \sin \left(w_{n}\left(\frac{x}{l}\right)\right) e^{-\frac{B}{2} x} f(x) e^{-\frac{B}{2} x} e^{B x} d x . \\
& =\frac{2}{l} \int_{0}^{l} \sin \left(\frac{n \pi x}{l}\right) f(x) d x .
\end{aligned}
$$

\section{Illustrative example}

Let the following mathematical problem be considered:

$$
\begin{aligned}
& u_{t}=u_{x x}+u_{x}-u, \\
& u(0, t)=0, u(2, t)=0, \\
& u(x, 0)=-\sin (\pi x) e^{-\frac{1}{2} x}
\end{aligned}
$$

whose solution is given in the following form:

$$
u(x, t)=-e^{-\frac{1}{2} x} \sin (\pi x) e^{-\left(\pi^{2}+\frac{5}{4}\right) t}
$$

where $0 \leq x \leq 2,0 \leq t \leq T$.

Example 1. Now the following time fractional form of above problem is taken into account:

$$
\begin{aligned}
& { }_{1}^{P} D_{t}^{\alpha} u(x, t)=u_{x x}(x, t)+u_{x}(x, t)-u(x, t), \\
& u(0, t)=u(2, t)=0 \\
& u(x, 0)=\sin (\pi x) e^{-\frac{1}{2} x}
\end{aligned}
$$


where $0<\alpha<1,0 \leq x \leq 2,0 \leq t \leq T$. It is clear from Eq. (20) that the solution of the above problem can be obtained in the following form:

$$
u(x, t ; \alpha)=\sum_{n=1}^{\infty} A_{n} e^{-\frac{1}{2} x} \sin \left(w_{n}\left(\frac{x}{2}\right)\right) \exp \left(-\frac{1+\frac{w_{n}^{2}+1}{4}+1-\alpha}{\alpha} t\right)
$$

where $w_{n}=n \pi$. Plugging $t=0$ into the general solution (28) and making it equal to the initial condition (27), we have

$$
-\sin (\pi x) e^{-\frac{1}{2} x}=\sum_{n=1}^{\infty} A_{n} e^{-\frac{1}{2} x} \sin \left(w_{n}\left(\frac{x}{2}\right)\right) .
$$

The coefficients $A_{n}$ for $n=1,2,3, \ldots$ are determined by the help of the inner product as follows:

$$
\begin{aligned}
A_{n} & =\frac{2}{l}<\sin \left(w_{n}\left(\frac{x}{2}\right)\right) e^{-\frac{1}{2} x},-\sin (\pi x) e^{-\frac{1}{2} x}> \\
& =\int_{0}^{2} \sin \left(w_{n}\left(\frac{x}{2}\right)\right) e^{-\frac{1}{2} x}(-\sin (\pi x)) e^{-\frac{1}{2} x} e^{x} d x .
\end{aligned}
$$

For $n \neq 2, A_{n}=0 . n=2$ we get

$$
A_{2}=-\int_{0}^{2} \sin ^{2}(\pi x) d x=-1 .
$$

Thus

$$
u(x, t ; \alpha)=e^{-\frac{1}{2} x} \sin (\pi x) \exp \left(-\frac{1+\frac{4 \pi^{2}+1}{4}+1-\alpha}{\alpha} t\right) .
$$

The accuracy of the obtained solution is checked by substituting $\alpha=1$ into (31), which leads to the solution of the problem (24).

Example 2. Now the following time fractional form of above problem is taken into consideration:

$$
\begin{aligned}
& { }_{2}^{P} D_{t}^{\alpha} u(x, t)=u_{x x}(x, t)+u_{x}(x, t)-u(x, t), \\
& u(0, t)=u(2, t)=0 \\
& u(x, 0)=\sin (\pi x) e^{-\frac{1}{2} x}
\end{aligned}
$$

where $0<\alpha<1,0 \leq x \leq 2,0 \leq t \leq T$. It is clear from Eq. (20) that the solution of the above problem can be obtained in the following form:

$$
u(x, t ; \alpha)=\sum_{n=1}^{\infty} A_{n} e^{-\frac{1}{2} x} \sin \left(w_{n}\left(\frac{x}{2}\right)\right) \exp \left(-\frac{1+\frac{4 \pi^{2}+1}{4}+1-\alpha^{2}}{\alpha^{2}} t\right) .
$$




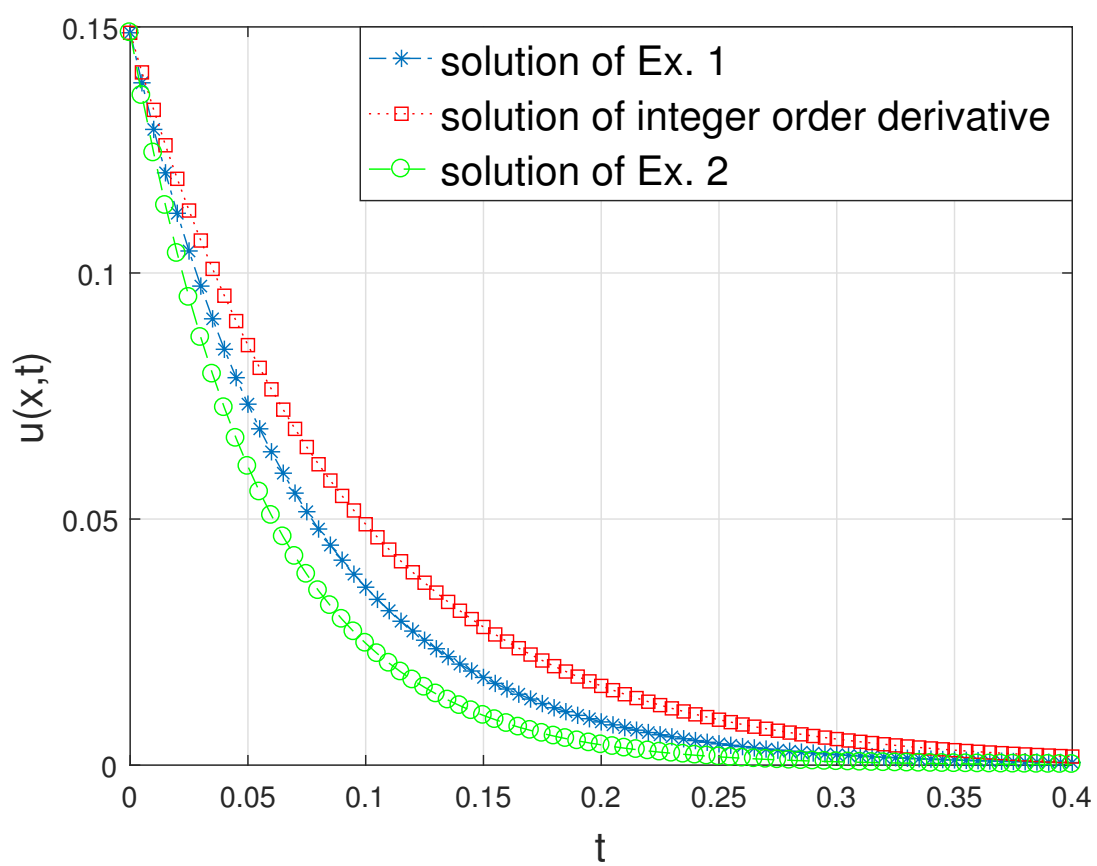

Fig. 1. The graphics of solutions for Ex. 1 and Ex. 2 in 2D at $x=0.1$ for $\alpha=0.8$

As in Example 1, after similar computations, the solution can be constructed as follows:

$$
u(x, t ; \alpha)=e^{-\frac{1}{2} x} \sin (\pi x) \exp \left(-\frac{1+\frac{4 \pi^{2}+1}{4}+1-\alpha^{2}}{\alpha^{2}} t\right) .
$$

The graphics of solutions for Ex.1, Ex. 2 and Problem (23) in 2D and 3D are given in Figures 1 and 2 respectively.

\section{Conclusion}

In this study, the analytic solution of time fractional diffusion problem including local fractional derivatives in one dimension is constructed analytically in Fourier series form. Taking the separation of variables into account, the solution is formed in the form of a Fourier series with respect to the eigenfunctions of a corresponding Sturm-Liouville eigenvalue problem.

Based on the analytic solution, we reach the conclusion that diffusion processes decays exponentially in time until initial condition is reached. As $\alpha$ tends to 0 , the rate of decaying increases. This implies that in the mathematical model for diffusion of the matter, which has a small diffusion rate, the value of $\alpha$ must be close to 0 . This model can account for various diffusion processes of various methods. 


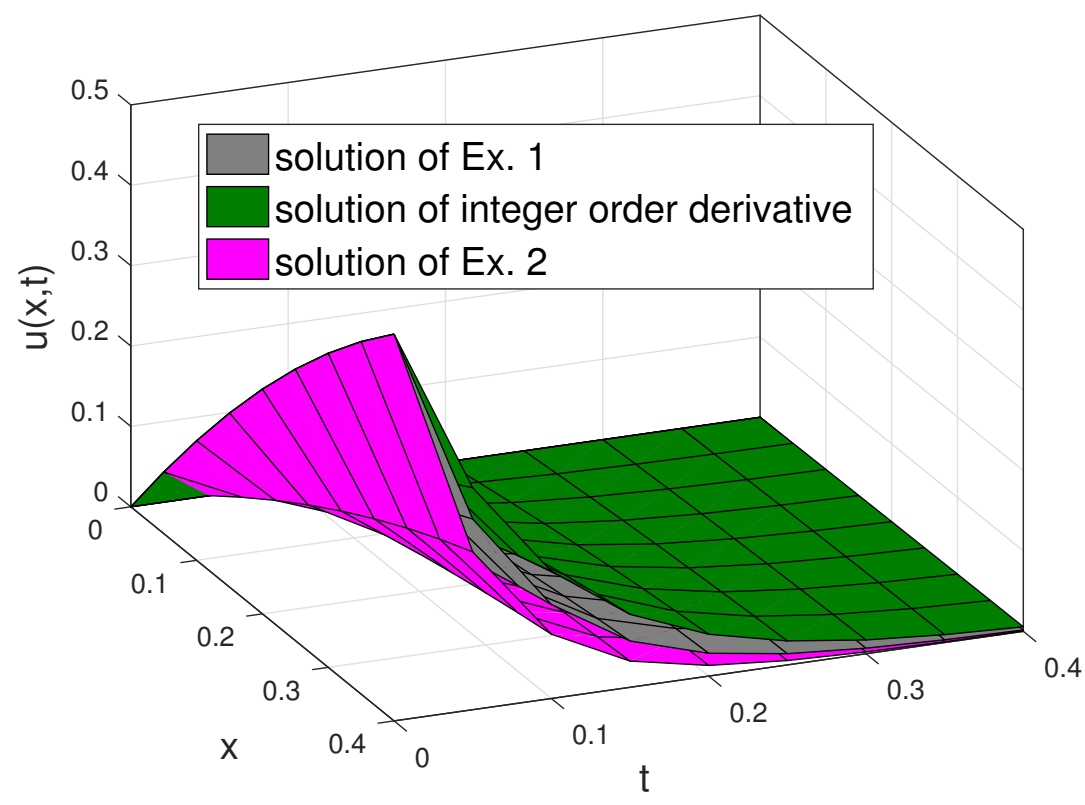

Fig. 2. The graphics of solutions for Ex. 1 and Ex. 2 in 3D for $\alpha=0.8$

\section{References}

[1] Baleanu, D., Fernandez, A., \& Akgul, A. (2020). On a fractional operator combining proportional and classical differintegrals. Mathematics, 8(360).

[2] Bisquert, J. (2005). Interpretation of a fractional diffusion equation with nonconserved probability density in terms of experimental systems with trapping or recombination. Physical Review E, $72,011109$.

[3] Sene, N. (2019). Solutions of fractional diffusion equations and Cattaneo-Hristov diffusion model. International Journal of Analysis and Applications, 17(2), 191-207.

[4] Aguilar, J.F.G., \& Hernández, M.M. (2014). Space-time fractional diffusion-advection equation with Caputo derivative. Abstract and Applied Analysis, 2014, Article ID 283019.

[5] Naber, M. (2004). Distributed order fractional sub-diffusion. Fractals, 12(1), 23-32.

[6] Nadal, E., Abisset-Chavanne, E., Cueto, E., \& Chinesta, F. (2018). On the physical interpretation of fractional diffusion. Comptes Rendus Mecanique, 346, 581-589.

[7] Zhang, W., \& Yi, M. (2016). Sturm-Liouville problem and numerical method of fractional diffusion equation on fractals. Advances in Difference Equations, 2016(217).

[8] Qureshi, S., Yusuf, A., \& Aziz, S. (2021). Fractional numerical dynamics for the logistic population growth model under Conformable Caputo: a case study with real observations. Physica Scripta, 96(11).

[9] Qureshi, S. (2020). Real life application of Caputo fractional derivative for measles epidemiological autonomous dynamical system. Chaos, Solitons \& Fractals, 134, 109744.

[10] Arqub, O.A., \& Shawagfeh, N. (2019). Application of reproducing kernel algorithm for solving Dirichlet time-fractional diffusion-Gordon types equations in porous media. Journal of Porous Media, 22(4), 411-434. 
[11] Arqub, O.A., \& Al-Smadi, M. (2020). An adaptive numerical approach for the solutions of fractional advection-diffusion and dispersion equations in singular case under Riesz's derivative operator. Physica A: Statistical Mechanics and its Applications, 540, 123257(1-13).

[12] Arqub, O.A., \& Al-Smadi, M. (2020). Numerical solutions of Riesz fractional diffusion and advection-dispersion equations in porous media using iterative reproducing kernel algorithm. Journal of Porous Media, 23(8), 783-804.

[13] Djennadi, S., Shawagfeh, N., \& Arqub, O.A. (2021). A fractional Tikhonov regularization method for an inverse backward and source problems in the time-space fractional diffusion equations. Chaos, Solitons \& Fractals, 150, 111127.

[14] Yusuf, A., Aliyu, A.I. \& Hashemi, M.S. (2018). Soliton solutions, stability analysis and conservation laws for the brusselator reaction diffusion model with time-and constant-dependent coefficients. The European Physical Journal Plus, 133(5), 1-11.

[15] Yusuf, A., \& Bayram, M. (2019). Invariant and simulation analysis to the time fractional Abrahams-Tsuneto reaction diffusion system. Physica Scripta, 94(12), 125005.

[16] Aliyu, A.I., İnç, M., Yusuf, A., \& Baleanu, D. (2018). Invariant subspace and lie symmetry analysis, exact solutions and conservation laws of a nonlinear reaction-diffusion Murray equation arising in mathematical biology. Journal of Advanced Physics, 7(2), 176-182.

[17] Günerhan, H. (2020). Analytical and approximate solution of two-dimensional convectiondiffusion problems. An International Journal of Optimization and Control: Theories \& Applications (IJOCTA), 10(1), 73-77.

[18] Cetinkaya, S., \& Demir, A. (2019). The analytic solution of time-space fractional diffusion equation via new inner product with weighted function. Communications in Mathematics and Applications, 10(4), 865-873.

[19] Cetinkaya, S., Demir, A., \& Kodal Sevindir, H. (2020). The analytic solution of sequential spacetime fractional diffusion equation including periodic boundary conditions. Journal of Mathematical Analysis, 11(1), 17-26.

[20] Cetinkaya, S., Demir, A., \& Kodal Sevindir, H. (2021). Solution of space-time-fractional problem by Shehu variational iteration method. Advances in Mathematical Physics, Article ID 5528928, (2021). 